\title{
Outcomes of pulpectomy in the primary dentition and age-related morphological changes in the root canal of primary molars: A retrospective study
}

\section{Guili Dou}

Peking University School and Hospital of Stomatology: Peking University School of Stomatology https://orcid.org/0000-0003-2184-6518

Bin Xia ( $\nabla$ summerinbeijing@vip.sina.com )

Peking University School and Hospital of Stomatology: Peking University School of Stomatology https://orcid.org/0000-0002-2801-5904

\section{Junxia Zhu}

Peking University School and Hospital of Stomatology: Peking University School of Stomatology

\section{Dandan Wang}

Peking University School and Hospital of Stomatology: Peking University School of Stomatology

\section{Yijiao Zhao}

Peking University School and Hospital of Stomatology: Peking University School of Stomatology

\section{Zhipeng Sun}

Peking University School and Hospital of Stomatology: Peking University School of Stomatology

\section{Zhanqiang Cao}

Peking University School and Hospital of Stomatology: Peking University School of Stomatology

\section{Research article}

Keywords: Cone-beam computed tomography, Primary teeth, Pulpectomy, Root canal morphology, Survival analysis

Posted Date: March 22nd, 2021

DOI: https://doi.org/10.21203/rs.3.rs-350568/v1

License: (a) (1) This work is licensed under a Creative Commons Attribution 4.0 International License. Read Full License 


\section{Abstract}

Objectives To investigate the factors influencing primary tooth survival after pulpectomy and to elucidate age-related morphological changes in the root canal of primary molars.

Materials and Methods This retrospective study included 592 primary anterior teeth and 583 primary molars after pulpectomy. Survival analysis was performed to determine the predictors of pulpectomy failure. Cone-beam computed tomography (CBCT) data of the maxillary first primary teeth from 63 children aged 3-8 years were collected, and Spearman's correlation and binary logistic regression were performed to assess age-related changes in canal morphology.

Results Periapical periodontitis, Vitape ${ }^{\circledR}$ filling, and non-general anesthesia treatment increased the failure risk of anterior primary teeth pulpectomy. Older age at treatment increased the primary molar pulpectomy failure risk. On CBCT analysis, the ratio of canal length to root length for the mesiobuccal $(\mathrm{MB})$, distobuccal $(\mathrm{DB})$, and palatal $(\mathrm{P})$ roots negatively correlated with age $(P<0.05)$. The ratio between the root canal wall area with inner diameter lesser than $0.15 \mathrm{~mm}$ and the whole root canal wall area $\left(\mathrm{R}_{.15}\right)$ at plane $\mathrm{D}$ of the MB canals positively correlated with age $(P<0.05)$. The number of discontinuous canals $\left(\mathrm{N}_{\mathrm{dis}}\right)$ for the $\mathrm{MB}$ and $\mathrm{DB}$ roots increased with age and the canal area at plane $\mathrm{D}$ for MB and $\mathrm{P}$ canals negatively correlated with age $(P<0.05)$.

Conclusions Older age at treatment for primary molar pulpectomy and periapical lesions, non-general anesthesia treatment, and Vitapex ${ }^{\circledR}$ filling for anterior primary teeth pulpectomy may predict a higher failure risk. Age-related morphological changes in the primary molar root canal were evident and could be a reason for treatment failure.

\section{Background}

Dental caries is a prevalent chronic disease in children, especially in developing countries. In China, the fourth national oral epidemiological investigation showed that the prevalence of dental caries in children aged 5 years was $71.9 \%$ and that the mean decayed-missing-filled teeth ( $\mathrm{dmft}$ ) index was 4.24 [1], both of which were considerably higher than those 10 years earlier $(66.0 \%, \mathrm{dmft}=3.5)$ [2]. Dental caries in the primary dentition progresses rapidly, often affecting the pulp within a short duration. In China, the incidence of periapical periodontitis is high in primary teeth [3]. The American Association of Pediatric Dentistry (AAPD) recommends pulpectomy as an elective treatment for periapical periodontitis in primary teeth and regards pulpectomy as a final treatment to retain primary teeth with diffuse irreversible pulpitis or pulp necrosis [4].

Previously, research has largely focused on primary tooth pulpectomy, mainly on filling materials [5-7], irrigant [8], and instrumentation methods [9, 10], reporting success rates between $56 \%$ and $97 \%$. However, a short follow-up period or the small sample size of these studies have led to varying success rates, as 
mentioned above. A large number of primary teeth treated with pulpectomy were evaluated in this study, with a follow-up of more than two years for most teeth.

It is imperative for operators to be familiar with the canal morphology of primary teeth to perform a successful pulpectomy. Research indicates that the canal morphology of permanent teeth is significantly different at different ages [11, 12]. Primary teeth exist in the mouth for a much shorter time than permanent teeth. Whether the canal system of primary teeth changes with age, and whether these changes influence the success of pulpectomy is still unknown. Owing to physiological absorption, it is impossible to obtain complete primary teeth of different ages for micro-CT or other in vitro analysis. The application and development of cone-beam computed tomography (CBCT) in stomatology provides a new method for studying the canal morphological characteristics of primary teeth. Through CBCT, it is possible to unravel the complete morphology of the canal in vivo. This technique has already been applied in the morphological analysis of permanent teeth $[13,14]$. In this study, CBCT was used to observe and measure the canal system of primary molars.

The aims of this study are as follows:

1) To study the long-term survival rate of pulpectomy in primary teeth and to analyse the potential factors influencing its success.

2) To study the age-related changes in the root canal system of primary molars using CBCT.

\section{Materials And Methods}

This retrospective study was approved by the Ethics Committee of Peking University School and Hospital of Stomatology (approval number: PKUSSIRB-201949122).

\section{Primary teeth pulpectomy}

\subsection{Participants}

The study participants were selected among healthy children below the age of 9 years, who received pulpectomy of the primary teeth in the Department of Pediatric Dentistry at Peking University Hospital of Stomatology. The inclusion criteria were as follows:

1) Teeth treated because of irreversible pulpitis or pulp necrosis, with or without periodontitis, for which radiographic examination showed no involvement of the permanent successor and minimal or no root resorption.

2) Teeth that had not undergone trauma.

3) Teeth that underwent regular postoperative examination with follow-up time $\geq 1.5$ years.

4) Teeth for which complete records were available. 
All pulpectomies were completed in compliance with the AAPD guidelines[4]. A six-month visit interval was recommended.

\subsection{Data collection}

The following information from an electronic medical record system (Beijing Jiahe Meikang Information Technology, Beijing, China) of Peking University Hospital of Stomatology was collected:

1. Demographic characteristics.

2. Information obtained from the first visit, including the date, rank of attending doctor (intern or expert), tooth position (anterior or posterior), treatment method (under general anesthesia (GA) or not [GA or non-GA]), periapical lesion (yes or no), clinical and radiographic manifestations, root filling material (iodoform zinc oxide paste[15] or Vitapex ${ }^{\circledR}$ [calcium hydroxide and iodoform paste, Neo-Dental, Tokyo, Japan]), and crown restoration material (Resin Filling, Preformed Metal Crown [PMC], or Glass lonomer Cement [GIC]).

3. Information obtained during follow-up, including following visit dates, chief complaints, and clinical and radiographic examination. If the tooth was already missing, the date of loss and the associated symptoms were recorded.

\subsection{Clinical and radiological evaluation}

The pulpectomy was considered clinically successful in the absence of pain, abnormal mobility, gingival pathology, and severe crown restoration defects necessitating root canal retreatment or extraction, in relation to the tooth. If not, it was labelled as clinical failure.

If the radiographic examination revealed a decrease in size or disappearance of the initial periapical lesions within 6 months[4], with no new appearances of periapical lesions and/or pathological root resorption, the treatment was classified as a radiological success; otherwise it was classified as a radiological failure.

The interventional outcome was defined as a success only if the treatment exhibited both clinical and radiological success. In addition, premature loss and delayed root resorption of primary teeth after treatment were classified as failure[4]. The primary teeth were evaluated for premature loss or delayed root resorption by comparison with the contralateral teeth and/or other adjacent teeth without pulp treatment. If there were no contralateral teeth and adjacent teeth without pulp treatment, it was estimated based on the development stage of the permanent successor.

All periapical films involved in this study were re-examined. Radiographic examinations were independently performed by two pediatric dentists. Cohen's kappa statistic showed excellent reproducibility between the two investigators, with a measurement agreement of 0.85 and intra-examiner reliability (over two weeks) of 0.87 . 


\section{Root canal morphology analysis}

\subsection{Data collection}

Data of CBCTs, which were originally conducted for diagnosis, treatment, or regular examinations, were retrieved from the Department of Radiology at the Peking University Hospital of Stomatology from March 2015 to November 2019. Radiographic images of systemically healthy children aged 3-8 years, were selected. The maxillary first primary molars of these children meeting the following criteria were included:

1. no abnormality in the crown and root morphology;

2. no pulpal involvement and periapical lesions;

3. had not undergone pulpotomy, pulpectomy or PMC restoration;

4. the development of the roots was complete, without internal or external absorption;

5. satisfactory image quality.

CBCT images were acquired using the 3D Accuitomo type F17 (Morita, Kyoto, Japan) at 80-90 kV and 5 $\mathrm{mA}$. The voxel and slice thicknesses were both $0.125 \mathrm{~mm}$. All CBCT exposures were performed by a licenced radiologist strictly in accordance with the manufacturer's recommended protocol.

\subsection{Measurements}

The CBCT scan data were saved in DICOM format and transferred to Mimics 17.0 (Materialise Technologies, Leuven, Belgium). Several points and values were defined as below, to aid with the measurement of each root and canal (Fig. 1a).

Point A: the lowest point of the outer surface of the root furcation.

Point B: the anatomical root tip.

Point C: the point where the canal disappeared towards the root tip for the first time.

$L_{R}$ : length of the root measured from points $A$ to $B$ along the tooth axis.

$\mathrm{L}_{C}$ : length of the canal measured from points $A$ to $C$ along the tooth axis.

Plane D: canal cross-section, $1 / 3 * L_{R}$ away from point $A$ along the tooth axis.

If the root canal cavity reappeared after disappearing toward the root tip, it was recorded as a discontinuous canal, as shown in Fig. 1a. The $L_{C}$ and $L_{R}$ were measured.

The canal cross-section at plane D was reconstructed. First, an appropriate threshold for accurate segmentation of the canal cavity was selected for each canal. The threshold value was determined as follows. We first measured the grey value range of the lip in the CBCT at three different positions and 
calculated the difference between the minimum and maximum grey values for each position. Then, the mean of the three differences was calculated and recorded as " $d$ ". The minimum grey value of the canal image was recorded as " $c$ ", and d plus c was " $p$ ", which was recorded as the reference threshold value of canal. Following this, the reference grey value " $p$ " was slightly adjusted for improved accuracy according to the operator's judgement.

After determination of a proper canal threshold, a three-dimensional (3D) model of the canal section at plane D was generated and measured, as shown in Fig. 1b. The detailed procedure is listed in Fig. 2. STL (stereo lithography) is a 3D graphics file format for rapid prototyping manufacturing.

The following outcome variables were calculated and recorded:

1) $L_{C} / L_{R}$ : Ratio of the canal length and root length.

2) $\mathrm{N}_{\text {dis }}:$ The number of discontinuous root canals.

3) $R_{.15}$ : The ratio of the root canal wall area with an inner diameter lesser than $0.15 \mathrm{~mm}$ and the total root canal wall area at plane $\mathrm{D}$.

4) Area of canal at plane D, measured using Image $J$ (National Institutes of Health, Bethesda, USA)

Before starting the study, 69 canal cross-section samples not included in this study were used to assess the consistency of image segmentation with the index of mean intersection over union (MloU). The MloU was 0.81 .

\section{Statistical analysis}

Statistical analyses were conducted using SPSS version 20.0 (SPSS Inc., Chicago, IL, USA). The survival rate of teeth after pulpectomy was determined using the Kaplan-Meier method. Univariate and multivariate analyses were performed using Cox regression analysis. Variables with $P<0.1$ in the univariate analysis were included in the multivariate analysis to be explored as possible risk factors. Factors previously reported to influence success were also included. The variables included in the Cox analysis were age, rank of attending doctor, treatment method, presence of periapical lesion, root canal filling material, and the crown restoration material. The Wald test (Backward: Wald) method was used, and the significance level of the selected and excluded variables was 0.05 .

The normality of data from CBCT was tested using the Kolmogorov-Smirnov one-sample test. The Spearman correlation coefficients between $R_{.15}, L_{C} / L_{R}$, area of canal at plane $D$, and age were calculated, and the linear regression coefficient between $\mathrm{N}_{\text {dis }}$ and age was calculated by binary logistic regression. The significance level was set at $P<0.05$.

\section{Results}




\section{Primary teeth pulpectomy}

Up to February 2019, 494 children (261 boys and 233 girls) and 1175 primary teeth were included in the study. The mean chronological age was 4.3 years, ranging from 1.4 to 8.5 years. The mean follow-up time was 998 days, ranging from 119 to 1871 days. The basic information on primary teeth interventions and the results of the univariate analysis results are listed in Table 1.

Table 1 Results of the univariate analysis in the study

\begin{tabular}{|c|c|c|c|c|}
\hline Co-variable & Level & $\mathrm{n}$ & $\%$ & $\begin{array}{l}\text { Univariate } \\
P \text {-value }\end{array}$ \\
\hline Overall & - & 1175 & 100 & - \\
\hline \multirow[t]{2}{*}{ Sex } & Boys & 626 & 53 & 0.892 \\
\hline & Girls & 549 & 47 & \\
\hline Age (continuous data) & - & & - & $<0.001^{*}$ \\
\hline \multirow[t]{2}{*}{ Tooth type } & Anterior & 592 & 50 & $<0.001^{*}$ \\
\hline & Posterior & 583 & 50 & \\
\hline \multirow[t]{2}{*}{ Rank of attending doctor } & Intern & 226 & 19 & $<0.001^{*}$ \\
\hline & Expert & 949 & 81 & \\
\hline \multirow[t]{2}{*}{ Treatment method } & Non-GA & 450 & 38 & $<0.001^{*}$ \\
\hline & GA & 725 & 62 & \\
\hline \multirow[t]{2}{*}{ Periapical lesion } & No & 847 & 72 & $0.004^{*}$ \\
\hline & Yes & 328 & 28 & \\
\hline \multirow[t]{2}{*}{ Root canal filling material } & Iodoform zinc oxide paste & 870 & 74 & 0.511 \\
\hline & Vitapex ${ }^{\circledR}$ & 305 & 26 & \\
\hline \multirow[t]{3}{*}{ Crown restoration material } & $\mathrm{RF}$ & 816 & 70 & $0.010^{*}$ \\
\hline & GIC & 51 & 4 & \\
\hline & PMC & 308 & 26 & \\
\hline
\end{tabular}

*Statistically significant $(P<0.05)$; GA, general anesthesia; GIC, glass ionomer cement; PMC, preformed

metal crown; RF, resin filling.

All included pulpectomies were divided into two groups: primary anterior teeth interventions and primary molar interventions. By the end of February 2019, 246 (41.6\%) primary anterior teeth interventions and $363(62.3 \%)$ primary posterior teeth interventions met the criteria for failure. Kaplan-Meier analysis showed that the median survival time for primary anterior teeth was 990 days and that for primary 
posterior teeth was 772 days. The cumulative survival rates of primary anterior teeth at 24 months and 30 months were $72.3 \%$ and $58.2 \%$ and that of primary molars were $57.1 \%$ and $37 \%$, respectively. Anterior teeth suffered less treatment failure than posterior teeth $(P<0.001)$.

The results of the multivariate Cox proportional hazards regression analysis are shown in Table 2. For primary anterior teeth, the failure risk of pulpectomy with GA was significantly lower than that of pulpectomy with no-GA. Treatment for teeth with periapical lesions and Vitapex ${ }^{\circledR}$ filling were more likely to fail $(P<0.05)$. For posterior primary teeth, age and the crown restoration material had a statistically significant impact on the failure risk of pulpectomy (Table 2). The failure risk of pulpectomies increased with age, and the hazard ratio (HR) was $1.185(95 \% \mathrm{Cl}, 1.082-1.297)(P<0.001)$. Interventions with $\mathrm{GIC}$ restorations were more likely to fail $(P<0.05)$.

Table 2 Results of multivariate Cox regression analysis of primary teeth after pulpectomy

\begin{tabular}{|c|c|c|c|c|c|}
\hline \multirow[t]{2}{*}{ Co-variable } & \multirow[t]{2}{*}{ Subgroup } & \multicolumn{2}{|c|}{$P$-valueHR } & \multicolumn{2}{|c|}{ 95\% CI for HR } \\
\hline & & & & Lower & Upper \\
\hline \multicolumn{6}{|l|}{ Anterior primary } \\
\hline \multicolumn{6}{|l|}{ teeth } \\
\hline \multirow[t]{2}{*}{ Treatment method } & Non-GA & & 1 & & \\
\hline & GA & 0.022 & 0.72 & 40.548 & 0.955 \\
\hline \multirow{2}{*}{ Periapical lesion } & No & & 1 & & \\
\hline & Yes & 0.010 & 1.44 & 61.093 & 1.914 \\
\hline Root canal filling & \multicolumn{2}{|l|}{ Iodoform zinc oxide paste } & \multicolumn{3}{|l|}{1} \\
\hline material & Vitapex ${ }^{\circledR}$ & 0.012 & 1.39 & 31.077 & 1.803 \\
\hline \multicolumn{6}{|l|}{ Posterior primary } \\
\hline \multicolumn{6}{|l|}{ molar } \\
\hline Age & - & 0.000 & 1.18 & 51.082 & 1.297 \\
\hline \multirow[t]{4}{*}{ Restoration materials } & & 0.028 & & & \\
\hline & $\mathrm{RF}$ & & 1 & & \\
\hline & GIC & 0.049 & 1.51 & 31.002 & 2.285 \\
\hline & $\mathrm{PMC}$ & 0.231 & 0.87 & 10.695 & 1.092 \\
\hline
\end{tabular}

GA, general anesthesia; GIC, glass ionomer cement; HR, hazard ratio; PMC, preformed metal cement; RF, resin filling.

\section{CBCT analysis of root canal morphology}

In total, 63 children ( 15 girls and 48 boys) were enrolled, involving 115 maxillary first primary molars. The average age was 5.9 years. All teeth were divided into six age groups, and the distribution of the children across these groups was as follows: $3-4$ years: $11 ; 4-5$ years: $30 ; 5-6$ years: $17 ; 6-7$ years: $20 ; 7-8$ years: 18; and 8-9 years: 19 . 
We found a positive correlation between $\mathrm{R}_{.15}$ of $\mathrm{MB}$ roots and age $\left(\mathrm{r}_{\mathrm{s}}=0.326, P<0.001\right)$, indicating that the canal diameter decreased with age. However, a similar result was not obtained for DB and $P$ roots. $L_{C} / L_{R}$ for $M B, D B$, and $P$ roots were negatively correlated with age (Fig. 3), suggesting a decrease in the canal length. The area of canal at plane $\mathrm{D}$ for $\mathrm{MB}$ and $\mathrm{P}$ roots diminished with age $(P<0.05)$ (Table 3$)$.

Table 3 Spearman's correlation between $R_{.15}, L_{C} / L_{R}$, area of canal at plane $D$, and age

\begin{tabular}{|c|c|c|c|c|c|c|c|c|c|}
\hline & \multicolumn{3}{|c|}{$\mathrm{R}_{.15}$} & \multicolumn{3}{|c|}{$\mathrm{L}_{\mathrm{C}} / \mathrm{L}_{\mathrm{R}}$} & \multicolumn{3}{|c|}{ Area of canal } \\
\hline & $\mathrm{MB}$ & DB & $\mathrm{P}$ & MB & DB & $\mathrm{P}$ & MB & DB & $\mathrm{P}$ \\
\hline$r_{s}$ & 0.326 & 0.103 & 0.116 & -0.349 & -0.405 & -0.207 & -0.343 & -0.171 & -0.371 \\
\hline$P$ & $0.000^{*}$ & 0.273 & 0.219 & $<0.001^{*}$ & $<0.001^{*}$ & $0.003^{*}$ & $<0.001^{*}$ & 0.066 & $<0.001^{*}$ \\
\hline
\end{tabular}

*Statistically significant $(P<0.05) ; \mathrm{R}_{.15}$, the ratio of the root canal wall area with an inner diameter lesser than $0.15 \mathrm{~mm}$ and the total root canal wall area at plane $\mathrm{D} ; \mathrm{L}_{\mathrm{C}} / \mathrm{L}_{\mathrm{R}}$, ratio of the canal length and root length; $\mathrm{DB}$, distobuccal root; $\mathrm{MB}$, mesiobuccal root; $\mathrm{P}$, palatal root.

Binary logistic regression analysis revealed that the occurrence of discontinuous canals for MB and DB roots increased significantly with age (Table 4).

Table 4 Results of binary logistic analysis for $\mathrm{N}_{\text {dis }}$ and age

\begin{tabular}{cllllll}
\hline Variables & $B$ & $P$-value & OR & \multicolumn{2}{c}{$95 \%$ CI for OR } \\
\cline { 5 - 6 } & & & & Lower & Upper \\
\hline $\mathrm{N}_{\text {dis }}$ & MB & 0.527 & $0.000^{*}$ & 1.693 & 1.267 & 2.262 \\
& DB & 0.352 & $0.005^{*}$ & 1.423 & 1.113 & 1.818 \\
$\mathrm{P}$ & 0.379 & 0.065 & 1.461 & 0.977 & 2.186 \\
\hline
\end{tabular}

*Statistically significant $(P<0.05)$; $\mathrm{N}_{\text {dis }}$, the number of discontinuous root canals; $\mathrm{B}$, unstandardized coefficient; CI, confidence interval; DB, distobuccal root; $\mathrm{MB}$, mesiobuccal root; OR, odds ratio; $\mathrm{P}$, palatal root.

\section{Discussion}

In this study, the survival rate of primary anterior teeth 24 months after pulpectomy was $72.3 \%$ and that of primary posterior teeth was $57.1 \%$, which was similar to the findings of previous studies $(63.3 \%-$ 78.5\%)[16-20]. The survival rate of primary anterior teeth was significantly higher than that of primary molars. This distinction may be due to a difference in the root canal morphology. Most anterior primary teeth have a single canal and few lateral branches or root tip bifurcations[21], but different forms of the accessory root canal are common in primary molars. Morabito et al. observed 30 primary molars under a scanning electron microscope and found that 21 of them had an accessory root canal at the bottom of 
the pulp chamber, in which pulp tissue and necrotic tissue were observed[22]. Therefore, primary anterior and posterior teeth were analysed independently in this study.

Among primary anterior teeth, the intervention failure risk of teeth with periapical lesions was significantly higher than that of teeth without periapical lesions. It is impossible to remove all pathogenic bacteria in the periapical tissue through root canal preparation, and residual bacteria can increase the postoperative failure risk. Chen et al. also reported worse prognosis of pulpectomy in primary teeth with periapical lesions[23]. However, interventions in primary molars with periapical lesions had a similar survival rate to teeth without periapical lesions. As mentioned before, the canal system of primary molars is more complicated than that of primary anterior teeth, and canal debridement is difficult. It is speculated that there is more residual infection in the primary molar canal system than in the root canals of anterior teeth, which can also cause recurrence of periapical periodontitis. In anterior teeth, non-GA treatment and Vitape ${ }^{\circledR}$ pulpectomy has a lower survival rate, which was consistent with findings of other studies[24, 25].

For primary molars, we found that the treatment at older ages was more likely to fail than that at a younger age, and the difference is statistically significant. This finding has never been reported previously. According to previous studies[12, 26, 27], permanent teeth show age-related changes, and secondary dentine deposition may change the root canal diameter or lead to severe canal calcification, increasing the difficulty of root canal preparation. In primary molars, similar age-related changes were also reported by Amano et al., who indicated that the volume ratio between the pulp chamber and the crown is larger in the primary dentition than in the mixed dentition[28]. Ahmed suggested that it is necessary to study the relationship between canal morphology and pulpectomy failure[29]. Therefore, it is hypothesised that age-related changes in primary molars may affect the outcome of pulpectomy.

The variable $R_{.15}$ was calculated to determine the difficulty of the $15 \mathrm{~K}$-file entering the canal. In this study, $R_{.15}$ increased and the canal area at plane $D$ of the MB canal decreased with age, indicating a decrease in the canal diameter. Similarly, Ge et al. studied the primary molars of children aged 3-8 years and found that the root canal orifice area in the mesial root decreased with age $(P<0.01)[30]$. Furthermore, we observed that most MB canal cross-sections were oval or flattened in the coronal-third of the root, and the canal diameter decreased rapidly with age (Fig. 4). The presence of an oval canal and a smaller canal diameter are challenging for mechanical and chemical canal instrumentation, resulting in larger areas of the dentine wall being untouched[31]. However, $\mathrm{R}_{.15}$ in DB and P canals were not found to correlate with age in this study. This is because most DB canal diameters were smaller than $0.15 \mathrm{~mm}$ or showed a complete calcification at plane $D$ at a young age in this study. In contrast to DB canals, for $P$ canals, nearly all diameters at plane $D$ were larger than $0.15 \mathrm{~mm}$ for all ages, but the canal area at plane $\mathrm{D}$ reduced significantly with age, reflecting the age-related changes in $\mathrm{P}$ canals.

Calcification in the apical third of the canal is reflected by the variables $L_{C} / L_{R}$ and $N_{\text {dis. }}$. The morphological analysis showed that $\mathrm{L}_{C} / \mathrm{L}_{R}$ decreased and $\mathrm{N}_{\text {dis }}$ increased significantly with age. Acquired dentine formation causes a decrease in the inner canal diameter, even partial or complete locking of the 
canal, resulting in length shortening and canal interruption. However, canal calcification in the apical third is usually incomplete, and in some canals, the lumen could still be observed below the calcification. Compared with the continuous root canal, it is more difficult to clean those discontinuous ones. In the case of primary anterior teeth, owing to the large canal diameter and the simple canal system, age-related acquired dentine formation has minimal impact on canal morphology. This may be the reason why the survival rate in primary anterior teeth did not change with age.

There are some limitations to this study. First, the inconsistency between radiographic and clinical manifestations makes it difficult to detect periapical diseases on time. However, in children, the potential damage caused by multiple radiographic evaluations should be carefully considered to determine if the risk-benefit ratio. Second, this is a retrospective study based on existing case records, in which there may be a recording inaccuracy. In order to avoid false records, we also checked the X-ray film when the records were examined. Third, the variables used in this study were static-observation CBCT indices, and the evaluation of the difficulty of root canal preparation was partly based on the diameter of the root canal, which cannot fully represent the actual situation of mechanical preparation. We hope that new technology can be invented to simulate the mechanical preparation process in the future, so the agerelated changes can be estimated more accurately.

\section{Conclusions}

The survival rate of primary anterior teeth was significantly higher than that of primary molars. For primary anterior teeth, the absence of periapical lesions, GA, and iodoform zinc oxide paste filling may improve the survival rate; for primary molars, a younger age at treatment may predict more successful treatment results. Root canal changes with age were evident, which may be one of the reasons for the failure of primary molar pulpectomy. These findings are reminders of the necessity of treating primary anterior teeth and primary molars differently and indicate directions to improve the success of pulpectomy in primary molars.

\section{Abbreviations}

CBCT: cone-beam computed tomography; MB: mesiobuccal; DB: distobuccal; P: palatal; GA: general anesthesia; PMC: preformed metal crown; GIC: glass ionomer cement; 3D: three-dimensional; STL: stereo lithography; MloU: mean intersection over union囚dmft: decayed-missing-filled teeth; AAPD: American Association of Pediatric Dentistry.

\section{Declarations}

\section{Acknowledgements}

The authors thank Xueying Li, from Peking University First Hospital, for help with the statistical analyses and the Wiley editing team for their language editing services. 


\section{Authors' contributions}

Bin Xia and Guili Dou have made substantial contributions to all the process of the study. Junxia Zhu, Dandan Wang, Zhipeng Sun, Zhanqiang Cao and Yijiao Zhao have made substantial contributions to the collection, analysis and interpretation of data. All authors have read and approved this manuscript.

\section{Funding}

This project was supported by grant Capital's Funds for Health Improvement and Research (Grant number: 2020-2-4105).

\section{Availability of data and materials}

The datasets used and/or analysed during the current study are available from the corresponding author on reasonable request.

\section{Ethics approval and consent to participate}

This retrospective study was conducted in accordance with the tenets of the Declaration of Helsinki for research involving human subjects, and approved by the Ethics Committee of Peking University School and Hospital of Stomatology (approval number: PKUSSIRB-201949122).

\section{Competing interests}

The authors declare that they have no competing interests.

\section{References}

1. Wang X: Report of the fourth national oral health epidemiology investigation. Beijing, China: People's Medical Publishing House; 2018.

2. Qi XQ: Report of the third national oral health epidemiology investigation. Beijing, China: People's Medical Publishing House; 2008.

3. Xia B, Qin M, Han Y, Zhang S. [Children stomatology outpatient treatment requirements analysis and countermeasures]. Beijing Da Xue Xue Bao Yi Xue Ban. 2013;45(1):92-6.

4. AAPD Council On Clinical Affairs. Pulp therapy for primary and immature permanent teeth. Pediatr Dent. 2018;40(6):343-51.

5. Pramila R, Muthu MS, Deepa G, Farzan JM, Rodrigues SJ. Pulpectomies in primary mandibular molars: a comparison of outcomes using three root filling materials. Int Endod J. 2016;49(5):413-21.

6. Nakornchai S, Banditsing P, Visetratana N. Clinical evaluation of 3Mix and Vitapex as treatment options for pulpally involved primary molars. Int J Paediatr Dent. 2010;20(3):214-21.

7. Trairatvorakul C, Chunlasikaiwan S. Success of pulpectomy with zinc oxide-eugenol vs calcium hydroxide/iodoform paste in primary molars: a clinical study. Pediatr Dent. 2008;30(4):303-8. 
8. Verma N, Sangwan P, Tewari S, Duhan J. Effect of different concentrations of sodium hypochlorite on outcome of primary root canal treatment: a randomized controlled trial. J Endod. 2019;45(4):35763.

9. George S, Anandaraj S, Issac JS, John SA, Harris A. Rotary endodontics in primary teeth - A review. Saudi Dent J. 2016;28(1):12-7.

10. Azar MR, Safi L, Nikaein A. Comparison of the cleaning capacity of Mtwo and Pro Taper rotary systems and manual instruments in primary teeth. Dent Res J (Isfahan). 2012;9(2):146-51.

11. Thomas RP, Moule AJ, Bryant R. Root canal morphology of maxillary permanent first molar teeth at various ages. Int Endod J. 1993;26(5):257-67.

12. Morse DR, Esposito JV, Schoor RS. A radiographic study of aging changes of the dental pulp and dentin in normal teeth. Quintessence Int. 1993;24(5):329-33.

13. Silva EJ, Nejaim Y, Silva AV, Haiter-Neto F, Cohenca N. Evaluation of root canal configuration of mandibular molars in a Brazilian population by using cone-beam computed tomography: an in vivo study. J Endod. 2013;39(7):849-52.

14. Zhang R, Wang H, Tian YY, Yu X, Hu T, Dummer PM. Use of cone-beam computed tomography to evaluate root and canal morphology of mandibular molars in Chinese individuals. Int Endod $\mathrm{J}$. 2011;44(11):990-9.

15. Zhao DH, Xie LH, Liu H, Qin M, Han R, Zheng LG. Formulation optimization of iodoform paste for root canal filling of deciduous teeth. Zhongguo Yao Xue Za Zhi. 2017;52(2):135-9.

16. Primosch RE, Ahmadi A, Setzer B, Guelmann M. A retrospective assessment of zinc oxide-eugenol pulpectomies in vital maxillary primary incisors successfully restored with composite resin crowns. Pediatr Dent. 2005;27(6):470-7.

17. Casas MJ, Kenny DJ, Johnston DH, Judd PL, Layug MA. Outcomes of vital primary incisor ferric sulfate pulpotomy and root canal therapy. J Can Dent Assoc. 2004;70(1):34-8.

18. Chandra SP, Chandrasekhar R, Uloopi KS, Vinay C, Kumar NM. Success of root fillings with zinc oxide-ozonated oil in primary molars: preliminary results. Eur Arch Paediatr Dent. 2014;15(3):191-5.

19. Mortazavi M, Mesbahi M. Comparison of zinc oxide and eugenol, and Vitapex for root canal treatment of necrotic primary teeth. Int J Paediatr Dent. 2004;14(6):417-24.

20. Howley B, Seale NS, McWhorter AG, Kerins C, Boozer KB, Lindsey D. Pulpotomy versus pulpectomy for carious vital primary incisors: randomized controlled trial. Pediatr Dent. 2012;34(5):112-9.

21. Salama FS, Anderson RW, McKnight-Hanes C, Barenie JT, Myers DR. Anatomy of primary incisor and molar root canals. Pediatr Dent. 1992;14(2):117-8.

22. Morabito A, Defabianis P. A SEM investigation on pulpal-periodontal connections in primary teeth. ASDC J Dent Child. 1992;59(1):53-7.

23. Chen Y, Li H, Li M, Yang L, Sun Q, Chen K. Analysis of survival and factors associated with failure of primary tooth pulpectomies performed under general anaesthesia in children from South China. Int $\mathrm{J}$ Paediatr Dent. 2020;30(2):225-33. 
24. Zhou F, Xia B, Zhang S, Ma WL, Xiao YM, Ge LH. [Comparison of long-term dental treatment effects of children treated under general anesthesia and passive restraint]. Zhonghua Kou Qiang Yi Xue Za Zhi. 2017;52(2):96-102.

25. Chen X, Liu X, Zhong J. Clinical and radiographic evaluation of pulpectomy in primary teeth: a 18months clinical randomized controlled trial. Head Face Med. 2017;13(1):12.

26. Kiefner P, Connert T, Elayouti A, Weiger R. Treatment of calcified root canals in elderly people: a clinical study about the accessibility, the time needed and the outcome with a three-year follow-up. Gerodontology. 2017;34(2):164-70.

27. Morse DR, Esposito JV, Schoor RS, Williams FL, Furst ML. A review of aging of dental components and a retrospective radiographic study of aging of the dental pulp and dentin in normal teeth. Quintessence Int. 1991;22(9):711-20.

28. Amano M, Agematsu $H$, Abe S, Usami A, Matsunaga S, Suto $K$, Ide Y. Three-dimensional analysis of pulp chambers in maxillary second deciduous molars. J Dent. 2006;34(7):503-8.

29. Ahmed HM. Anatomical challenges, electronic working length determination and current developments in root canal preparation of primary molar teeth. Int Endod J. 2013;46(11):1011-22.

30. Ge L, Zheng S, Zhang T. Morphologic study of the root canal orifices in human mandibular primary molars. Xian Dai Kou Qiang Yi Xue Za Zhi. 2002;16(4):363-5.

31. Paque F, Balmer M, Attin T, Peters OA. Preparation of oval-shaped root canals in mandibular molars using nickel-titanium rotary instruments: a micro-computed tomography study. J Endod. 2010;36(4):703-7.

\section{Figures}



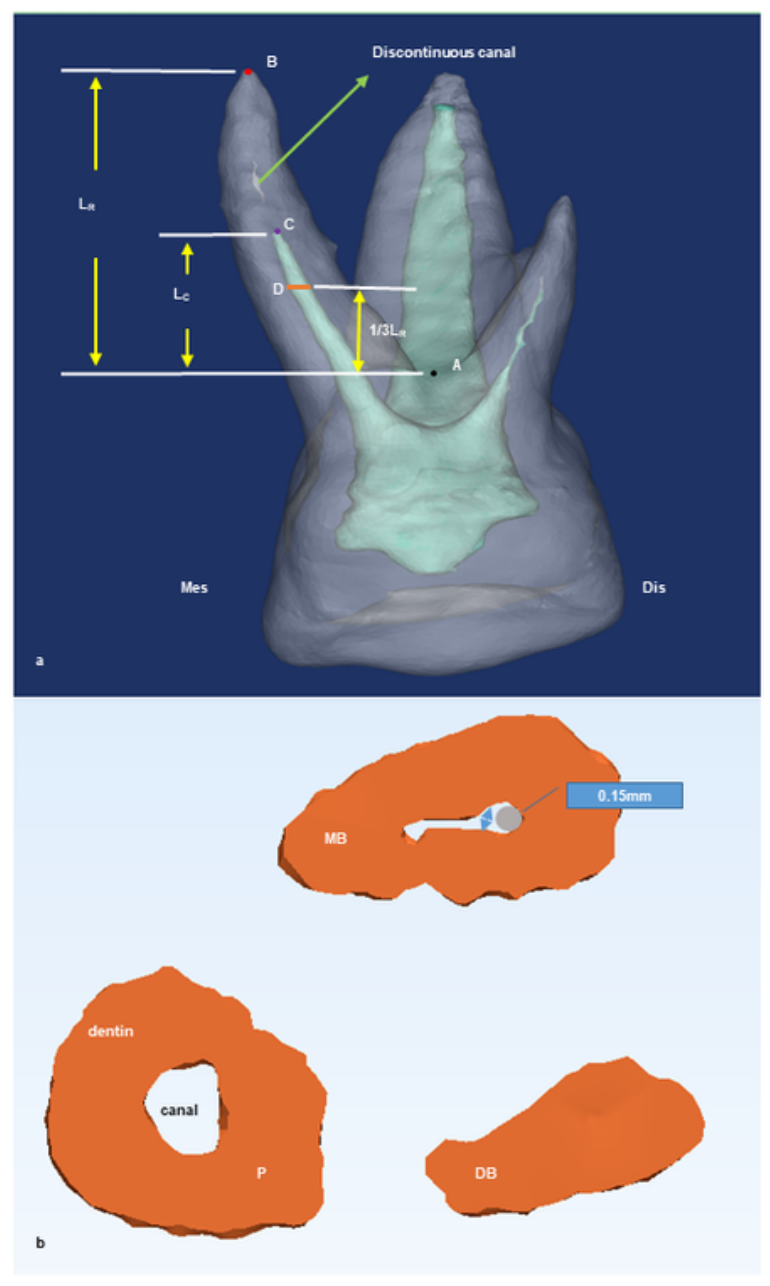

\section{Figure 1}

Representative three-dimensional (3D) reconstruction of the CBCT scan. a: 3D reconstruction of the CBCT scans. Points $A, B$, and $C$, plane $D$, and lengths $L C, L R$ are represented; $b$ : The canal cross sections at plane $D$ of the MB root, DB root, and $P$ root are reconstructed. The grey circle represents the tip of the $15 \#$ $\mathrm{K}$-file, and the blue arrows represent inner canal diameter at different positions. $A$, the lowest point of the outer surface of the root furcation; B, the anatomical root tip; $\mathrm{C}$, the point where the canal disappeared 
towards the root tip for the first time; DB, distobuccal; Dis, distal surface; LC, length of the canal measured from points $A$ to $C$ along the tooth axis; $L R$, length of the root measured from points $A$ to $B$ along the tooth axis; MB, mesiobuccal; Mes, mesial surface; P, palatal; Plane $D$, canal cross-section, $1 / 3 \star$ LR away from point $A$ along the tooth axis

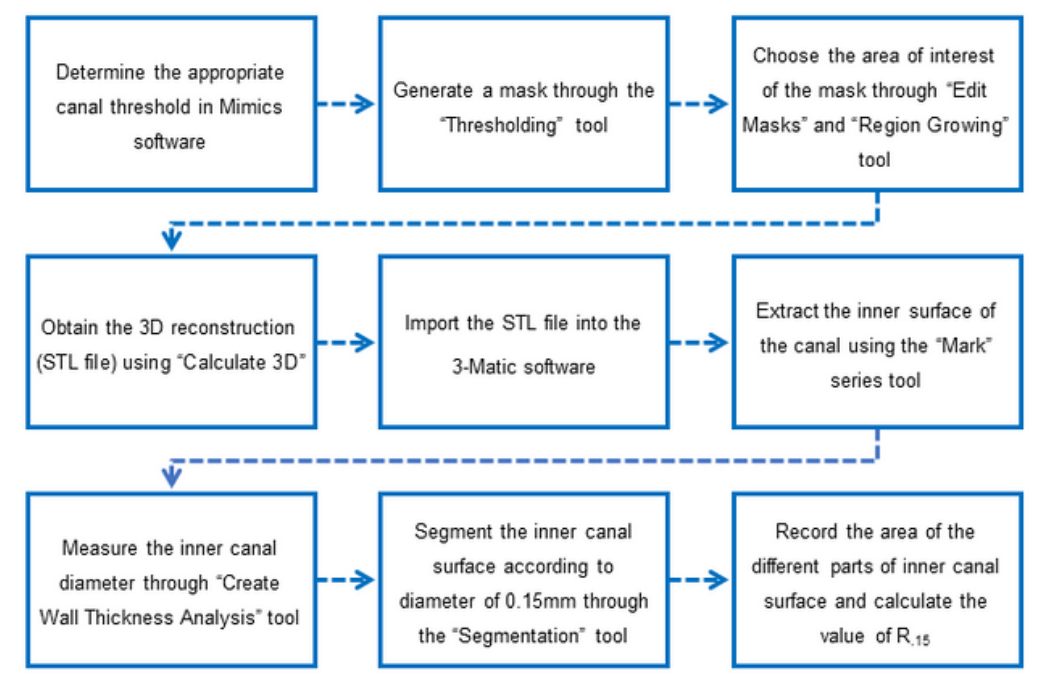

Figure 2

Detailed procedure of 3D reconstruction and canal measurement at plane $\mathrm{D}$. 3D, three-dimensional; Plane $D$, canal cross section, $1 / 3^{*} \mathrm{LR}$ [length of the root measured along the tooth axis from point $A$ (lowest point of the outer surface of the root furcation) to point $B$ (anatomical root tip)] away from point $A$ along the tooth axis; R15, the ratio of the root canal wall area with an inner diameter lesser than $0.15 \mathrm{~mm}$ and the total root canal wall area at plane D; STL, stereo lithography 


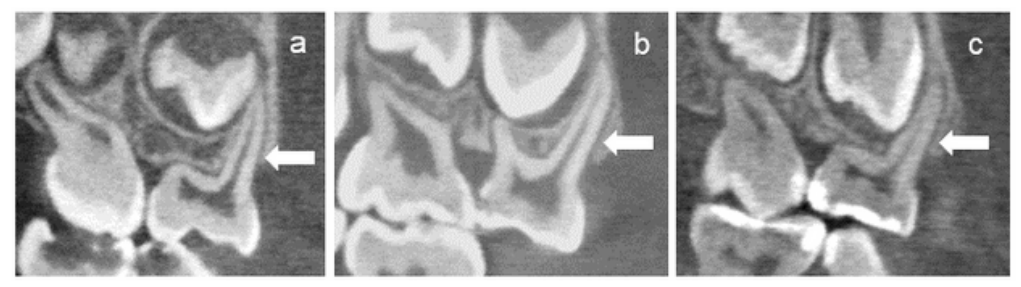

\section{Figure 3}

The canal length of the left maxillary first primary molar reduces relative to root with age. (a) MB canal from a 4-year-old child; (b) MB canal from a 6-year-old child; (c) MB canal from an 8-year-old child. LC/ LR decreases with age. LC, length of the canal along the tooth axis measured from the lowest point of the outer surface of the root furcation to the point where the canal disappeared towards the root tip for the first time; LR, length of the root measured along the tooth axis from the lowest point of the outer surface of the root furcation to the anatomical root tip; MB, mesiobuccal 

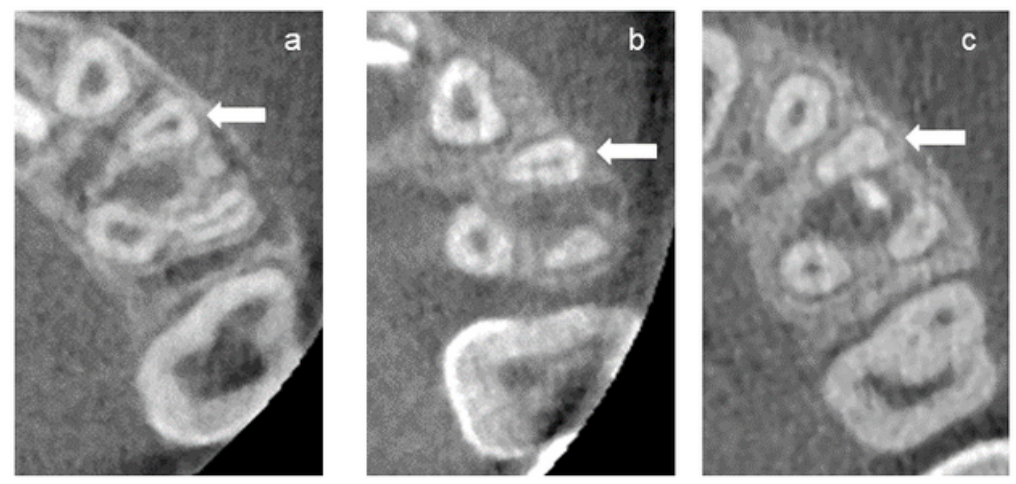

\section{Figure 4}

The canal diameter of the left maxillary first primary molar becomes smaller with age. (a) MB canal from a 3-year-old child; (b) MB canal from a 6-year-old child; (c) MB canal from an 8-year-old child. From a to c, the lumen of MB canals is decreased. MB, mesiobuccal 